\title{
Eotaxin and monocyte chemoattractant protein-1 in chronic eosinophilic pneumonia
}

\author{
H. Tateno*, H. Nakamura*, N. Minematsu*, K. Amakawa*, T. Terashima*, S. Fujishima*, \\ A.D. Luster", C.M. Lilly", K. Yamaguchi*
}

Eotaxin and monocyte chemoattractant protein-1 in chronic eosinophilic pneumonia. $H$. Tateno, H. Nakamura, N. Minematsu, K. Amakawa, T. Terashima, S. Fujishima, A.D. Luster, C.M. Lilly, K. Yamaguchi. (C) ERS Journals Ltd 2001.

ABSTRACT: Chronic eosinophilic pneumonia (CEP) is characterized by chronic or recurrent pulmonary infiltrates with eosinophils, but the precise mechanism of eosinophil accumulation has not been fully elucidated. Eotaxin is one of the CC chemokines that selectively recruits eosinophils and contributes to the pathogenesis of allergic airway diseases including asthma, but its roles in pathogenesis of CEP have not been fully elucidated.

The authors measured concentrations of eotaxin and other $\mathrm{CC}$ chemokines, monocyte chemoattractant protein-1 (MCP-1), regulated on activation, normal T-cell expressed and secreted, macrophage inflammatory protein-1 $\alpha$, and the eosinophil activating Th2 cytokine interleukin (IL)-5 in bronchoalveolar lavage (BAL) fluid from CEP patients $(n=11)$, and compared these concentrations with those from control subjects $(\mathrm{n}=6)$.

The eotaxin $\left(904 \pm 203\right.$ versus $\left.29 \pm 7 \mathrm{pg} \cdot \mathrm{mL}^{-1}, \mathrm{p}=0.0001\right)$, MCP-1 $(194 \pm 57$ versus $\left.15 \pm 2 \mathrm{pg} \cdot \mathrm{mL}^{-1}, \mathrm{p}<0.05\right)$, and IL-5 $\left(7.8 \pm 2.0\right.$ versus $\left.2.7 \pm 0.6 \mathrm{pg} \cdot \mathrm{mL}^{-1}, \mathrm{p}<0.05\right)$ levels were significantly higher for cases with CEP in comparison to those serving as controls. Proportions of eosinophil and lymphocyte counts were greater in BAL fluid from CEP patients. Eotaxin and IL-5 levels correlated with the proportion of eosinophils in BAL fluid from CEP patients. MCP-1 correlated with the relative lymphocyte numbers.

In short, eotaxin, interleukin-5, and monocyte chemoattractant protein-1 levels were higher in the BAL fluid of CEP patients and these levels may contribute to eosinophil and lymphocyte recruitment and activation in the airways as found with this disorder. Eur Respir J 2001; 17: 962-968.
*Dept of Medicine, School of Medicine, Keio University, Tokyo, Japan and ${ }^{\#}$ Brigham and Women's and Massachusetts General Hospital, Harvard Medical School, Boston, MA, USA.

Correspondence: H. Nakamura, Cardiopulmonary Division, Dept of Medicine, School of Medicine, Keio University, 35 Shinanomachi, Shinjuku-ku, Tokyo 160-8582, Japan

Fax: 81333532502

Keywords: Bronchoalveolar lavage chronic eosinophilic pneumonia eotaxin

interleukin-5

interstitial lung disease

monocyte chemoattractant protein-1

Received: May 102000

Accepted after revision December 14 2000
Chronic eosinophilic pneumonia (CEP) is an idiopathic pulmonary disorder characterized by marked eosinophil accumulation in the airways. Patients with CEP have chronic clinical symptoms including fever, night sweats, and dyspnoea and $\sim 50 \%$ of the patients experience asthmatic symptoms. The prognosis is usually good as the radiographic infiltrates and symptoms are usually reduced by treatment with corticosteroids, but relapse is not unusual during cessation of corticosteroid treatment [1-3].

Eotaxin is one of the $\mathrm{CC}$ chemokines that selectively recruits eosinophils to tissues and has recently been reported to contribute to the pathogenesis of allergic airway inflammation including bronchial asthma [4-8]. Previous studies demonstrated that eotaxin and interleukin (IL)-5, the potent eosinophil-activating Th2 cytokine, collaborate to promote eosinophil recruitment into inflamed tissues in animal models [9]. In addition, increased production of various CC chemokines like eotaxin, regulated upon activation, normal T-cell expressed and secreted (RANTES), macrophage inflammatory protein (MIP)-1 $\alpha$, and monocyte chemoattractant protein (MCP)-1 has been demonstrated in allergic airway inflammation with eosinophils [10-12].
Since eosinophil accumulation is much more prominent in the lung parenchyma of CEP patients compared to those in asthmatics, $\mathrm{CC}$ chemokines active on eosinophils may contribute to the processes of eosinophil mobilization in CEP. KURASHIMA et al. [13] reported that the $\mathrm{CC}$ chemokine, RANTES, had higher levels in bronchoalveolar lavage (BAL) fluid obtained from CEP patients, but the roles of the eosinophil-selective CC chemokine eotaxin in CEP have not been fully elucidated. To determine whether eotaxin plays a significant role in the pathogenesis of CEP coordinating with other CC chemokines and IL-5, concentrations of eotaxin, RANTES, MIP- $1 \alpha$, MCP-1, and IL-5 were measured in BAL fluid harvested from CEP patients, and compared to those obtained from healthy control subjects and to those from patients with other interstitial lung diseases.

\section{Methods}

\section{Study populations}

Eleven patients with CEP (19-75-yr-olds, male/ female (M/F): 9/2, current smoker/exsmoker/nonsmoker 
(C/E/N) 2/6/3) were admitted to Keio University Hospital between 1991-1998. Inclusion criteria were patients with: 1) clinical symptoms persisting for $>2$ weeks (fever, weight loss, dry cough, and dyspnoea); 2) predominantly peripheral pulmonary infiltrates on chest radiographs; 3) increased eosinophil counts in BAL fluid (proportion of eosinophils $>10 \%$ ); and 4) the absence of predisposing causes such as a drug allergy, a parasite infection, vasculitis, or inhaled antigens $[1-3,13]$. No CEP patients had been treated with either corticosteroids or immunosuppressive agents when BAL was performed. Transbronchial lung biopsy (TBLB) was performed on eight patients with CEP and the pathological findings were consistent with CEP, while vasculitis, granuloma, and significant fibrosis were not observed. Nine patients with idiopathic pulmonary fibrosis (IPF) (37-75 yrs, M/F 7/2, $\mathrm{C} / \mathrm{E} / \mathrm{N} 2 / 4 / 3$ ) and eight with sarcoidosis (SAR) (24-47 yrs, M/F 4/4, C/E/N 4/1/3) were evaluated as disease controls in the current study. These patients were admitted to Keio University Hospital between 1997-1998. The IPF patients fulfilled all of the major diagnostic criteria and at least three of the four minor criteria described in the international consensus statement [14]. TBLB specimens obtained from the IPF patients suggested pulmonary fibrosis but had no features supporting other diseases. The SAR patients had abnormal findings on chest radiographs (stage I or II) and were diagnosed by TBLB specimens showing noncaseating epithelioid cell granulomas [15]. None of them had any history of inhaling inorganic materials known to cause granulomatous diseases. Six control subjects (CTL) (18-25 yrs, M/F 6/0, C/E/N 0/0/6) were healthy volunteers and exhibited neither respiratory symptoms nor abnormal chest radiograph findings. None of the subjects in the IPF, SAR, and CTL groups had been treated with either corticosteroids or immunosuppressive agents before the BAL procedures. Samples were collected after obtaining informed consent from each subject. The study was approved by the ethics committee at Keio University Hospital.

\section{Bronchoalveolar lavage}

BAL was performed in the involved lung segment or middle lobe. The bronchoscope was advanced into the involved segment and wedged; sterile normal saline was instilled three times in 50-mL aliquots. The recovered BAL fluid was centrifuged at $200 \times g$ and $4{ }^{\circ} \mathrm{C}$ for $5 \mathrm{~min}$, and cell-free supernatants were collected and frozen at $-70^{\circ} \mathrm{C}$ until use. The cell pellets were used for the differential counts on Wright-Giemsa-stained preparations.

\section{Measurement of chemokine and interleukin-5 concen-} trations

Concentrations of eotaxin in BAL fluids were measured by enzyme linked immunosorbent assay (ELISA) in duplicate as previously described [4, 5, 7, $8]$, with a detection limit of $15.6 \mathrm{pg} \cdot \mathrm{mL}^{-1}$. The concentrations of MCP-1, RANTES, IL-5, and MIP- $1 \alpha$ were measured in duplicate using ELISA kits from R\&D Systems (Minneapolis, MN, USA). The detection limits were $5.0,5.0,3.0$, and $7.0 \mathrm{pg} \cdot \mathrm{mL}^{-1}$, respectively. All measurements were performed using unconcentrated BAL fluids.

\section{Statistical analysis}

Data were expressed as mean \pm SEM. Analysis of variance was used to compare mean values. Simple linear regression analysis was performed to evaluate the correlation between concentrations of each chemokine or IL-5 in BAL supernatants and proportions of BAL cells. In addition, normalized multiple linear regression analysis was employed to assess the correlation between the various mediator concentrations and the BAL inflammatory cells. A p-value $<0.05$ was considered statistically significant.

\section{Results}

Characteristics of clinical manifestations in chronic eosinophilic pneumonia patients

Clinical features of CEP patients are summarized in table 1. Patient age ranged 19-75 yrs and the duration of symptoms before diagnosis ranged 2-36 weeks. The chronic, progressive illness did not improve after treatment with antibiotics for any of the patients, but significantly improved after corticosteroid treatment for eight patients. For three patients, the symptoms gradually lessened without the administration of steroids. The reason for this choice, i.e. no steroid treatment, was hyperglycaemia in one patient (case 9) and slow but spontaneous regression in two patients (cases 1 and 4). Previous asthmatic symptoms were confirmed for five patients. Eosinophil percentages of BAL fluids ranged $14.3-71.1 \%$. A peripheral blood eosinophilia level of $>6 \%$ was seen in eight cases.

\section{Bronchoalveolar cell differentials}

Total cell counts of BAL fluid from CEP patients were higher than those from the CTL group, but not in comparison with IPF and SAR (CEP: $11.6 \pm 3.5$ versus CTL: $2.7 \pm 0.5 \times 10^{5} \mathrm{~mL}^{-1}, \mathrm{p}<0.05$, IPF: $5.9 \pm 1.5$ $\times 10^{5} \mathrm{~mL}^{-}$, SAR: $\left.6.5 \pm 1.0 \times 10^{5} \mathrm{~mL}^{-1}\right)$. The proportion of eosinophils and lymphocytes in BAL fluid obtained from CEP, IPF, SAR, and CTL is shown in figure 1 . The eosinophil ratio was markedly higher in patients with CEP. The lymphocyte percentage was the highest for SAR and the second highest for CEP. The percentage of neutrophils in BAL fluid was the highest for IPF patients (CEP: $3.7 \pm 1.0$, IPF: $5.6 \pm 2.1$, SAR: $0.7 \pm 0.3$, CTL: $0.3 \pm 0.1 \%, \mathrm{p}<0.05$, IPF versus SAR or CTL).

Chemokine and interleukin-5 concentrations in bronchoalveolar lavage fluids

Eotaxin recovery was markedly augmented in the CEP group $\left(904 \pm 203 \mathrm{pg} \cdot \mathrm{mL}^{-1}\right)$ than in other groups 
Table 1. - Patient profiles for chronic eosinophilic pneumonia

\begin{tabular}{|c|c|c|c|c|c|c|c|c|}
\hline Case & Sex & $\begin{array}{l}\text { Age } \\
\text { yrs }\end{array}$ & $\begin{array}{l}\text { Duration* } \\
\text { weeks }\end{array}$ & Asthma & Relapse & $\begin{array}{c}\text { BALF } \\
\text { TCC } \times 10^{5} \cdot \mathrm{ml}^{-1}\end{array}$ & $\begin{array}{l}\text { BALF } \\
\text { Eo } \%\end{array}$ & $\begin{array}{c}\mathrm{PB} \\
\mathrm{Eo}^{0} \%\end{array}$ \\
\hline 1 & M & 37 & 8 & + & + & 5.9 & 14.3 & 24.0 \\
\hline 2 & M & 50 & 28 & - & + & 14.2 & 18.3 & 3.3 \\
\hline 3 & M & 73 & 8 & - & - & 5.6 & 62.1 & 19.6 \\
\hline 4 & M & 19 & 2 & - & - & 16.3 & 71.1 & 12.0 \\
\hline 5 & M & 58 & 8 & + & - & 5.8 & 36.5 & 33.1 \\
\hline 6 & M & 70 & 12 & + & - & 6.2 & 20.3 & 11.9 \\
\hline 7 & M & 52 & 12 & + & - & 10.2 & 22.6 & 36.8 \\
\hline 8 & M & 62 & 36 & - & + & 2.8 & 37.6 & 39.7 \\
\hline $9^{\#}$ & M & 75 & 4 & - & - & 7.1 & 27.3 & 18.4 \\
\hline 10 & $\mathrm{~F}$ & 39 & 28 & + & + & 8.8 & 29.1 & 3.8 \\
\hline $11^{\#}$ & $\mathrm{~F}$ & 57 & 3 & - & - & 44.4 & 50.0 & 2.0 \\
\hline \multicolumn{2}{|c|}{ Mean \pm SEM } & $53.8 \pm 5.1$ & $12.7 \pm 3.2$ & & & $11.6 \pm 3.5$ & $35.5 \pm 5.6$ & $18.6 \pm 4.0$ \\
\hline
\end{tabular}

M: male; F: female; BALF: bronchoalveolar lavage fluid; TCC: total cell count; Eo\%: percentage of eosinophils; PB: peripheral blood; ${ }^{\#}$ : patients with eotaxin levels lower than $30 \mathrm{pg} \cdot \mathrm{ml}^{-1} ;{ }^{*}$ : weeks before diagnosis.

(CTL: $29 \pm 7$, IPF: $161 \pm 17$, SAR: $149 \pm 21 \mathrm{pg} \cdot \mathrm{mL}^{-1}$ ) (fig. 2a). In two CEP patients, BAL eotaxin levels were $<30 \mathrm{pg} \cdot \mathrm{mL}^{-1}(\sim$ the mean value in the CTL group) despite the presence of eosinophil accumulation (fig. 2a). In contrast, MCP-1 levels were higher for the IPF $\left(354 \pm 69 \mathrm{pg} \cdot \mathrm{mL}^{-1}\right)$ and CEP $\left(194 \pm 57 \mathrm{pg} \cdot \mathrm{mL}^{-1}\right)$ groups than for the CTL group $\left(15 \pm 2 \mathrm{pg} \cdot \mathrm{mL}^{-1}\right)$ (fig. 2b). Although the concentrations of RANTES and MIP-1 $\alpha$ were lower than those of eotaxin and MCP-1 for CEP patients and no statistical difference was observed in the levels of both chemokines between CEP and CTL, their levels in the CEP group tended to be somewhat higher than those in the CTL group (RANTES: $30 \pm 14$ versus $1.2 \pm$ $0.6 \mathrm{pg} \cdot \mathrm{mL}^{-1}, \mathrm{p}=0.052$, MIP-1 $\alpha: 31 \pm 13$ versus $11 \pm 0.2, p=0.13$ ) (figs. $2 \mathrm{c}$ and $2 \mathrm{~d}$ ). The IL-5 levels detected in BAL fluid were relatively low, but they were significantly higher in the CEP group ( $7.8 \pm$ $\left.2.0 \mathrm{pg} \cdot \mathrm{mL}^{-1}\right)$ than in the SAR $\left(3.0 \pm 0.2 \mathrm{pg} \cdot \mathrm{mL}^{-1}\right)$ and CTL $(2.7 \pm 0.6)$ groups (fig. $2 \mathrm{e})$.

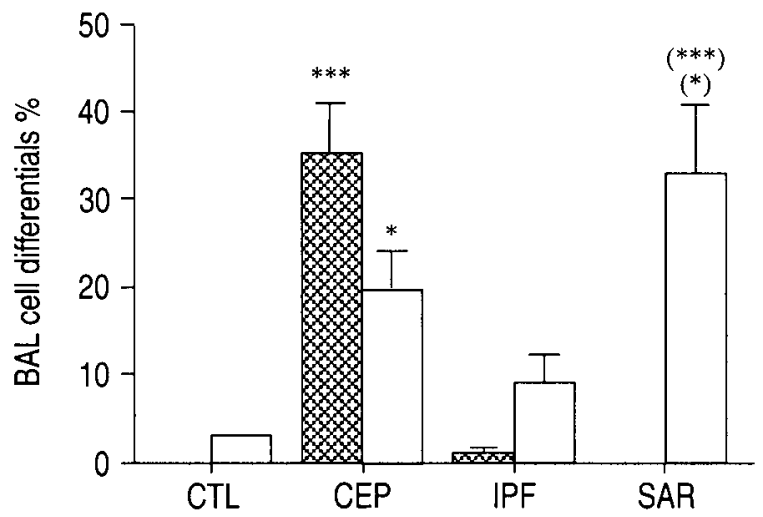

Fig. 1.- Percentage of eosinophils (伖) and lymphocytes ( $\square$ ) in bronchoalveolar lavage (BAL) fluid. Data represents the mean \pm SEM. CTL: control subjects $(n=6)$, CEP: chronic eosinophilic pneumonia $(n=11)$, IPF: idiopathic pulmonary fibrosis $(n=9)$; SAR: sarcoidosis $(\mathrm{n}=8)$. ***: $\mathrm{p}<0.001$ versus the other three groups, $(* * *): \mathrm{p}<0.001$ versus $\left.\mathrm{CTL} ;{ }^{*}\right): \mathrm{p}<0.05$ versus $\mathrm{CEP}$; $*: \mathrm{p}<0.05$ versus $\mathrm{CTL}$.
Correlation between the chemokine and interleukin-5 concentrations and bronchoalveolar eosinophils and lymphocytes

In order to elucidate the contribution of chemokines and IL-5 to leukocyte recruitment to the airspaces in CEP, correlation of the concentrations of these mediators with the percentage of eosinophils or lymphocytes in BAL fluid was evaluated using the data in CEP patients and CTL subjects. Eotaxin and IL-5 levels significantly correlated with the percentage of eosinophils by simple linear regression analysis (figs. 3a and 3b). MCP-1 distinctly correlated with relative lymphocyte numbers (fig. 3c). The correlation coefficients (r) and p-values between the other combinations in simple linear regression analysis are presented below. Eosinophil versus MCP-1: $r=0.34(p=0.18), M I P-1 \alpha$ : $\mathrm{r}=0.27(\mathrm{p}=0.29)$, RANTES: $\mathrm{r}=0.20(\mathrm{p}=0.44)$. Lymphocyte versus eotaxin: $r=0.11(p=0.68), M I P-1 \alpha$ : $r=0.03(p=0.90)$, RANTES: $r=0.15(p=0.57)$, IL-5: $\mathrm{r}=0.10(\mathrm{p}=0.70)$.

In multiple linear regression analysis, the coefficient of determination $\left(\mathrm{R}^{2}\right)$ between the relative eosinophil numbers and the concentrations of the five mediators in BAL fluid was $0.70(\mathrm{p}=0.01)$. Judging from the standard partial regression coefficients (SPRC), IL-5 and eotaxin levels had the dominant explanatory power for the relative eosinophil numbers in the BAL fluid, while those of the other three substances (MCP-1, MIP- $1 \alpha$, and RANTES) did not (table 2 ). The $\mathrm{R}^{2}$ value between the relative lymphocyte numbers and the concentrations of the five mediators in the BAL fluid was $0.71(p=0.01)$. Only the SPRC observed for MCP-1 was statistically significant (table 2). The results obtained from multiple linear regression analysis were qualitatively consistent with those from simple linear regression analysis.

\section{Discussion}

CEP is an idiopathic pulmonary disease characterized by marked lung parenchyma eosinophilia. Based on the selective effects of eotaxin on eosinophils and 

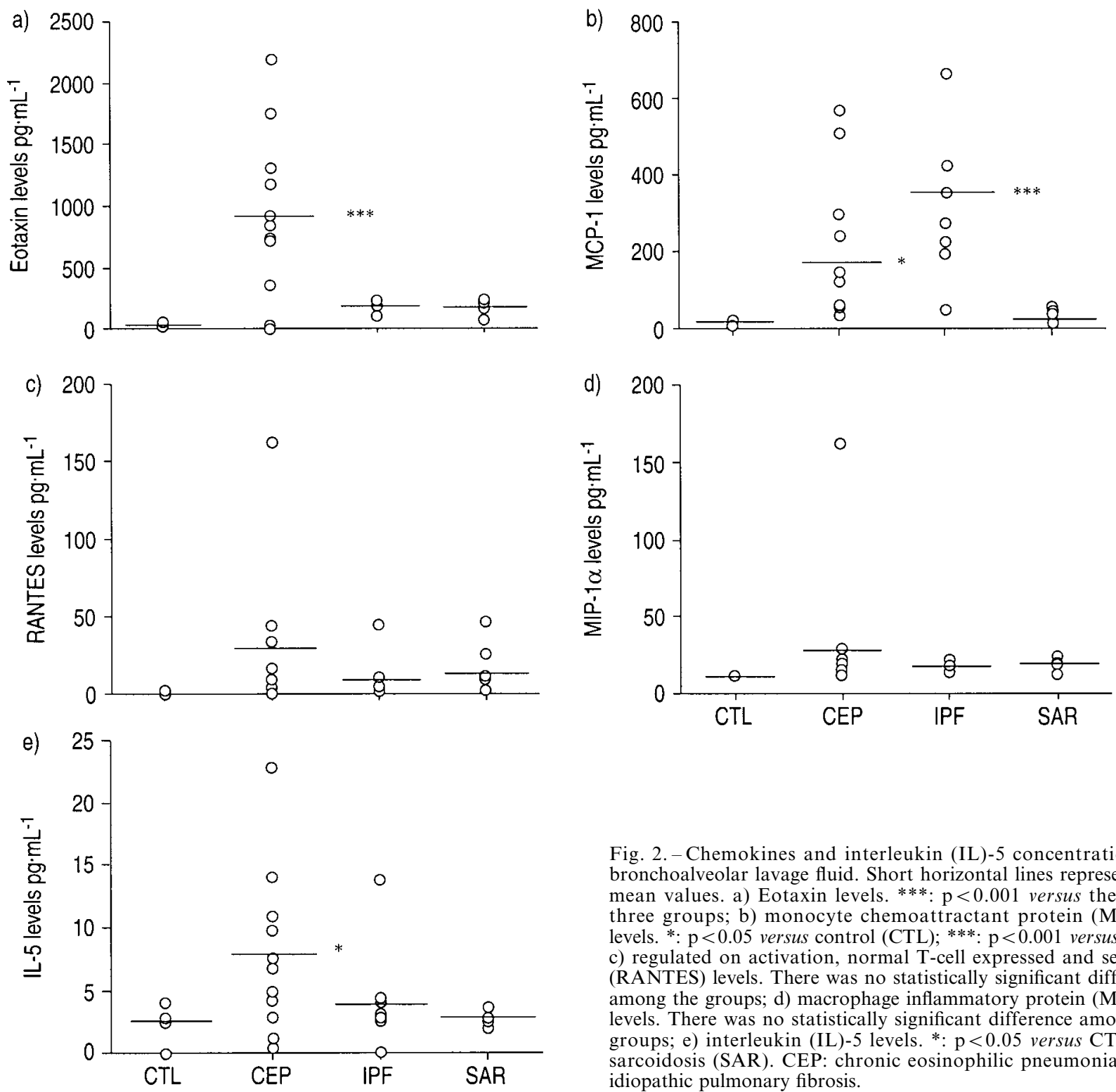

prominent eosinophil accumulation in CEP patients, the authors hypothesized that eotaxin is involved in the pathogenesis of CEP. Results suggest that eotaxin levels markedly increase and significantly correlate with the proportion of BAL eosinophils for the CEP patients, and the coordinated upregulation of eotaxin, IL-5, and MCP-1 may contribute to eosinophil and lymphocyte mobilization as observed in allergic airway inflammation including bronchial asthma [9-12].

Although $\sim 50 \%$ of CEP patients experience asthmatic symptoms, no specific allergens have been detected in CEP patients. In addition, BAL eosinophilia is not as prominent in asthma as it is in CEP (median; 3.9\%) [4] and eosinophil-related pulmonary infiltrates are not observed in asthmatics. Only a mild elevation of BAL eotaxin levels has been demonstrated in asthmatics compared to CEP (median; $53 \mathrm{pg} \cdot \mathrm{mL}^{-1}$ ) using the same eotaxin assay system as utilized in this study [4]. There were no significant differences in the

Fig. 2. - Chemokines and interleukin (IL)-5 concentrations in bronchoalveolar lavage fluid. Short horizontal lines represent the mean values. a) Eotaxin levels. $* * *: p<0.001$ versus the other three groups; b) monocyte chemoattractant protein (MCP)-1 levels. ${ }^{*}: \mathrm{p}<0.05$ versus control $(\mathrm{CTL}) ; * * * \mathrm{p}<0.001$ versus $\mathrm{CTL}$; c) regulated on activation, normal $\mathrm{T}$-cell expressed and secreted (RANTES) levels. There was no statistically significant difference among the groups; d) macrophage inflammatory protein (MIP)- $1 \alpha$ levels. There was no statistically significant difference among the groups; e) interleukin (IL)-5 levels. *: $\mathrm{p}<0.05$ versus $\mathrm{CTL}$ and sarcoidosis (SAR). CEP: chronic eosinophilic pneumonia; IPF: idiopathic pulmonary fibrosis.

chemokines and IL-5 concentrations in the BAL fluid between CEP patients who had experienced asthmatic symptoms and those who had not in the present study (data not shown). Th2 dominant lymphocyte activation is a characteristic of asthmatics, while both Th1 and Th2 cells may be involved in CEP [16]. Precise mechanisms accounting for the enhanced eosinophilrelated reaction with CEP are unknown, but the present study suggests that markedly higher eotaxin levels are involved in augmented eosinophilic inflammation. The presence of undetected allergen exposure or genetic differences cannot be excluded in the pathogenesis of CEP. In addition, it should be noted that there were two CEP patients with BAL eotaxin levels that were nearly undetectable despite the presence of significant eosinophil accumulation (table 1, fig. 2a) and that other factors are capable of recruiting eosinophils into the lung. These observations indicate that CEP may not be a single disorder in terms of 

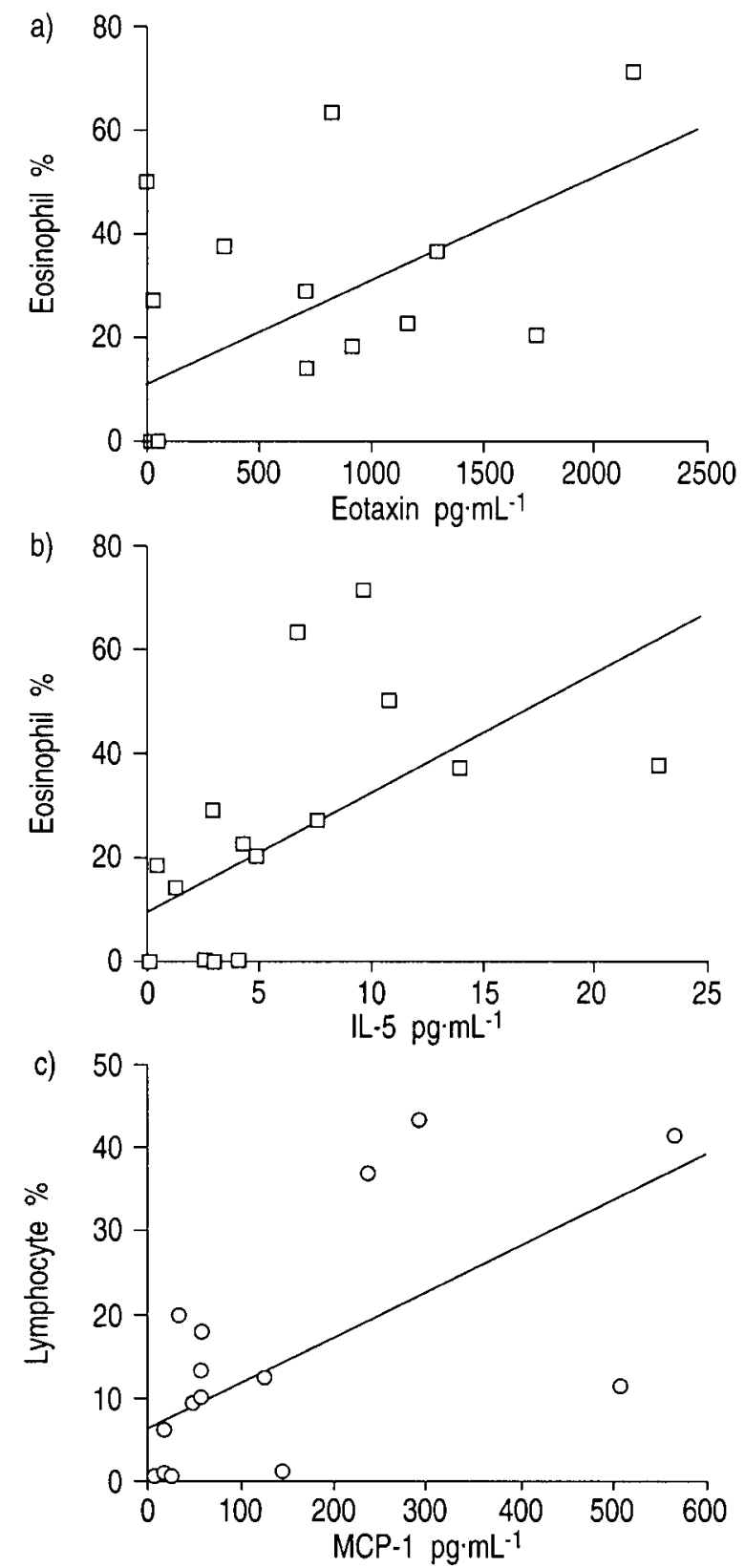

Fig. 3. - Simple linear regression analysis between the mediator concentrations and the proportions of eosinophils or lymphocytes in bronchoalveolar fluid. Data for chronic eosinophilic pneumonia $(\mathrm{CEP})(\mathrm{n}=11)$ and control $(\mathrm{CTL})(\mathrm{n}=6)$ subjects has been included in each figure. Correlations between a) \%eosinophil and eotaxin $(\mathrm{r}=0.582, \mathrm{p}=0.014)$; $\mathrm{b})$ interleukin (IL) -5 levels $(\mathrm{r}=0.575$, $\mathrm{p}=0.016)$; c) between \%lymphocyte and monocyte chemoattractant protein $(\mathrm{MCP})-1$ levels $(\mathrm{r}=0.662, \mathrm{p}=0.004)$ are presented.

eotaxin elaboration. The authors, however, found no clinical correlate of low eotaxin CEP in this study (table 1).

A previous report suggested that eotaxin production and eosinophil recruitment to the airway were diminished by the depletion of T-lymphocytes in a mouse model of allergic inflammation [17]. Both Th1 and Th2 cytokines enhanced cytokine-induced eotaxin expression in airway epithelial cells [4, 18]. In addition, activated helper T-cells had increased in number [19]
Table 2.-Multiple linear regression analysis between concentrations of the mediators and proportions of eosinophils and lymphocytes in bronchoalveolar lavage fluid

$\%$ Eosinophils \% Lymphocytes

$\overline{\text { SPRC t-values p-values }} \overline{\text { SPRC t-values p-values }}$

\begin{tabular}{lrrrrrl}
\hline Eotaxin & 0.460 & 2.52 & 0.028 & 0.239 & 1.31 & 0.134 \\
MCP-1 & 0.272 & 1.27 & 0.230 & 1.060 & 4.96 & 0.0004 \\
IL-5 & 0.741 & 2.43 & 0.033 & -0.136 & -0.45 & 0.664 \\
RANTES & 0.197 & 1.15 & 0.273 & 0.028 & 0.17 & 0.872 \\
MIP-1 $\alpha$ & -0.458 & -1.53 & 0.154 & -0.491 & -1.64 & 0.129 \\
\hline
\end{tabular}

SPRC: standard partial regression coefficient; MCP-1: monocyte chemoattractant protein-1; IL-5: interleukin-5; RANTES: regulated on activation, normal T-cell expressed and secreted; MIP-1 $\alpha$ : macrophage inflammatory protern- $1 \alpha$.

and both Th1 and Th2 cytokines have been observed in the airways of CEP patients [16]. These observations are consistent with the current findings, i.e. marked elevation of eotaxin levels is accompanied by lymphocyte accumulation and the elevation of IL-5 and MCP-1, both of which are potentially associated with T-lymphocyte activation. It is also possible that increased eotaxin in the airways may directly affect lymphocytes as CCR3 has been reported to be expressed on some Th2 cells [20]. No significant correlation was observed between eotaxin levels and the relative lymphocyte numbers in BAL fluid (table 2), but the contribution of eotaxin to recruitment and activation of Th2 lymphocytes in CEP cannot be excluded since subpopulations of lymphocytes were not discriminated in the present study. Although the main physiological role of MCP-1 is thought to be the activation of monocytic cells [21, 22], MCP-1 is also active on activated T-cells which express CCR2. The present findings suggest that MCP-1 contributes to lymphocyte accumulation in CEP (table 2 and fig. 3). RANTES can act on lymphocytes as well since it can bind to CCR1 and CCR5 in addition to CCR3 [21]. The current results indicate the presence of slightly elevated RANTES levels in CEP that seems to be consistent with previous research [13] (fig. 2b). Although the present study could not find significant correlations between RANTES or MIP- $1 \alpha$ levels and percentages of BAL eosinophils, it does not immediately mean lack of implication of these CC chemokines on eosinophils influx, since other factors not explored in this study may be implicated in the chemotaxis, for instance, the expression levels of CCR3 on eosinophils, the interaction between very late activation antigen-4 on eosinophils and vascular cell adhesion molecule-1 on endothelial cells. Furthermore, CC chemokines and IL-5 may act in a coordinated fashion [9].

It is interesting that the proportion of lymphocytes was higher in patients with sarcoidosis, but the MCP-1 levels were lower than those in CEP patients (figs. 1 and 2). These observations are attributed to the fact that Th1 dominant lymphocyte activation occurs without the aid of MCP-1 in sarcoidosis [23], while lymphocyte activation observed in CEP is similar to allergic inflammation including asthma in that the Th2 cytokine IL-5 and MCP-1 levels have increased [12]. In contrast, MCP-1 levels were higher in pulmonary 
fibrosis, but the lymphocyte counts were lower than those in CEP patients. It is to be elucidated why marked lymphocyte accumulation is not induced in the presence of abundant MCP-1 in pulmonary fibrosis. The differential status of activated alveolar macrophages may be responsible for the distinct differentials of BAL cells in CEP, sarcoidosis, and pulmonary fibrosis.

Although IL-5 levels were higher in CEP patients than those in the CTL group and significantly correlated with the percentage of eosinophils (figs. 2 and 3 , table 2), the levels in BAL fluid were relatively low when compared to those previously reported in patients with eosinophilic pneumonia [24, 25]. Previous reports described high IL-5 levels in BAL fluid, mainly in acute eosinophilic pneumonia. The authors also found a patient with acute eosinophilic pneumonia whose BAL IL-5 level was $>1 \mathrm{ng} \cdot \mathrm{mL}^{-1}$ (data not shown) applying the identical kit used for this study, indicating that the pathogenesis of acute eosinophilic pneumonia differs considerably from that of CEP in that a more remarkable but transient release of IL-5 may occur in acute eosinophilic pneumonia. In addition, IL-5 has been reported to be released from eosinophils per se besides Th2 lymphocytes [26]. These observations indicate a positive regulation of eosinophil through autocrine secretion of IL-5 in the airways of CEP patients.

In conclusion, a significant correlation between bronchoalveolar lavage eotaxin levels and eosinophils, and bronchoalveolar lavage monocyte chemoattractant protein-1 levels and lymphocytes in patients with chronic eosinophilic pneumonia has been demonstrated, suggesting that these chemokines contribute to the recruitment of inflammatory cells in the lung in chronic eosinophilic pneumonia.

\section{References}

1. Jederlinic PJ, Sicilian L, Gaensler EA. Chronic eosinophilic pneumonia: a report of 19 cases and a review of the literature. Medicine 1988; 67: 154-162.

2. Marchand E, Raynaud-Gaubert M, Lauque D, et al. Idiopathic chronic eosinophilic pneumonia: a clinical and follow-up study of 62 cases. Medicine 1998; 77 : 299-312.

3. Allen JN, Davis WB. Eosinophilic lung disease. Am J Respir Crit Care Med 1994; 150: 1423-1438.

4. Lilly CM, Nakamura H, Kesselman H, et al. Expression of eotaxin by human lung epithelial cells: induction by cytokines and inhibition by glucocorticoids. J Clin Invest 1997; 99: 1767-1773.

5. Lamkhioued B, Renzi PM, Abi-Younes S, et al. Increased expression of eotaxin in bronchoalveolar lavage and airways of asthmatics contributes to the chemotaxis of eosinophils to the site of inflammation. J Immunol 1997; 159: 4593-4601.

6. Ying S, Robinson DS, Meng Q, et al. Enhanced expression of eotaxin and CCR3 mRNA and protein in atopic asthma. Association with airway hyperresponsiveness and predominant colocalization of eotaxin mRNA to bronchial epithelial and endothelial cells. Eur J Immunol 1997; 27: 3507-3516.

7. Nakamura H, Weiss ST, Israel E, Luster AD, Drazen
JM, Lilly CM. Eotaxin and impaired lung function in asthma. Am J Respir Crit Care Med 1999; 160: 1952 1956.

8. Lilly CM, Woodruff PG, Camargo CA Jr, et al. Elevated plasma eotaxin levels in patients with acute asthma. J Allergy Clin Immunol 1999; 104: 786-790.

9. Wang J, Palmer K, Lotvall J, et al. Circulating, but not local lung, IL-5 is required for the development of antigen-induced airways eosinophilia. J Clin Invest 1998; 102: 1132-1141.

10. Gonzalo J, Lloyd CM, Wen D, et al. The coordinated action of $\mathrm{CC}$ chemokines in the lung orchestrates allergic inflammation and airway hyperresponsiveness. J Exp Med 1998; 188: 157-167.

11. Gonzalo JA, Lloyd CM, Kremer L, et al. Eosinophil recruitment to the lung in a murine model of allergic inflammation: the role of $\mathrm{T}$ cells, chemokines, and adhesion receptors. J Clin Invest 1996; 98: 2332-2345.

12. Holgate ST, Bodey KS, Janezic A, Frew AJ, Kaplan AP, Teran LM. Release of RANTES, MIP-1 alpha, and MCP-1 into asthmatic airways following endobronchial allergen challenge. Am J Respir Crit Care Med 1997; 156: 1377-1383.

13. Kurashima K, Mukaida N, Fujimura M, et al. A specific elevation of RANTES in bronchoalveolar lavage fluids of patients with chronic eosinophilic pnuemonia. Lab Invest 1997; 76: 67-75.

14. The American Thoracic Society, The European Respiratory Society. Idiopathic pulmonary fibrosis diagnosis and treatment. International consensus statement. Am J Respir Crit Care Med 2000; 161: 646-664.

15. The American Thoracic Society, The European Respiratory Society, The World Association of Sarcoidosis Other Granulomatous Disorders. Statement on sarcoidosis. Am J Respir Crit Care Med 1999; 160: 736-755.

16. Walker C, Bauer W, Braun RK, et al. Activated T cells and cytokines in bronchoalveolar lavages from patients with various lung diseases associated with eosinophilia. Am J Respir Crit Care Med 1994; 150: 1038-1048.

17. MacLean JA, Ownbey R, Luster AD. T cell-dependent regulation of eotaxin in antigen-induced pulmonary eosinophilia. J Exp Med 1996; 184: 1461-1469.

18. Garcia-Zepeda EA, Rothenberg ME, Ownbey RT, Celestin J, Leder P, Luster AD. Human eotaxin is a specific chemoattractant for eosinophil cells and provides a new mechanism to explain tissue eosinophilia. Nat Med 1996; 2: 449-456.

19. Mukae H, Kadota J, Kohno S, Matsukura S, Hara K. Increase of activated T-cells in BAL fluid of Japanese patients with bronchiolitis obliterans organizing pneumonia and chronic eosinophilic pneumonia. Chest 1995; 108: 123-128.

20. Sallusto F, Mackay CR, Lanzavecchia A. Selective expression of the eotaxin receptor CCR 3 by human T helper 2 cells. Science 1997; 277: 2005-2007.

21. Luster AD. Chemokines: chemotactic cytokines that mediate inflammation. N Engl J Med 1998; 338: 436445.

22. Luster AD, Rothenberg ME. Role of the monocyte chemoattractant protein and eotaxin subfamily of chemokines in allergic inflammation. $J$ Leukocyte Biol 1997; 62: 620-633.

23. Bergeron A, Bonay M, Kambouchner M, et al. Cytokine patterns in tuberculosis and sarcoid granulomas: 
correlations with histopathologic features of the granulomatous response. J Immunol 1997; 159: 3034 3043.

24. Okubo Y, Horie S, Hachiya $\mathrm{T}$, et al. Predominant implication of IL-5 in acute eosinophilic pneumonia: comparison with chronic eosinophilic pneumonia. Int Arch Allergy Immunol 1998; 116: 76-80.

25. Allen JN, Liao Z, Wewers MD, Altenberger EA,
Moore SA, Allen ED. Detection of IL-5 and IL-1 receptor antagonist in bronchoalveolar lavage fluid in acute eosinophilic pneumonia. J Allergy Clin Immunol 1996; 97: 1366-1374.

26. Lamkhioued B, Gounni AS, Aldebert D, et al. Synthesis of type 1 (IFN gamma) and type 2 (IL-4, IL-5, and IL-10) cytokines by human eosinophils. Ann N Y Acad Sci 1996; 796: 203-208. 